\title{
Control of the degradation of the mineral transformer oils hydrocarbon base
}

\author{
Marsel Sh. Garifullin ${ }^{1, *}$, Yuliya N. Solobodina ${ }^{1}$, Azat R. Bikzinurov ${ }^{1}$, Ruslan A. Giniatullin², and Alla G. Logacheva ${ }^{3}$
}

${ }^{1}$ Kazan State Power Engineering University, Department of Electric Power Systems and Networks, 420066 Kazan, Krasnoselskaya str., 51, Russia

${ }^{2}$ Kazan National Research Technological University, Department of Electric drives and electrical engineering, 420015, Kazan, Karl Marx str., 72, Russia

${ }^{3}$ Kazan State Power Engineering University, Department of Power supply of industrial enterprises, 420066 Kazan, Krasnoselskaya str., 51, Russia

\begin{abstract}
Currently, the degree of degradation of the oils hydrocarbon base remains outside the field of vision of operating services. The aim of this work was to analyse the process of regeneration of aged mineral transformer oils using adsorption cleaning. IR spectroscopy shows that the hydrocarbon composition of oils undergoes changes during operation. And those changes are cannot be restored. We propose the concept for the control of destruction degree of the mineral oils hydrocarbon base. The suggested criterion based on the IR method may be used when decision is making whether the transformer oil should be replaced with a new one.
\end{abstract}

\section{Introduction}

The average age of power oil-filled transformers all over the world exceeds 30 years and will steadily increase in the foreseeable future $[1,2]$. In this regard, the problem of ensuring the reliability of the operation of such equipment becomes more and more urgent every year. The service life of oil-filled transformers is primarily determined by the state of its insulation system, which is based on insulating paper and cardboard (solid insulation), as well as transformer oil. Transformer oil serves both as a liquid dielectric and a heat-dissipating medium.

At present, in the overwhelming majority of cases, mineral insulating oil produced from petroleum feedstock serves as transformer oil. Exposing the oil to unfavourable operating conditions leads to the formation of aging products in it. This not only impairs the performance of the oil, but also increases the rate of degradation of solid insulation.

If the quality of the oil does not meet the required standards, oil can be replaced with a new one. However, the main measures to restore its characteristics is the cleaning procedure or the complete regeneration of the oil. The cleaning process involves the restoration to the required values of standardized quality indicators such as acid number, moisture content, colour, $\operatorname{tg} \delta$, breakdown voltage, etc. If all undesirable components are removed from the oil and stabilized with appropriate additives, the oil is considered regenerated [3].

In addition to restoring operating parameters, the recovered oil visually (in colour and turbidity) acquires the same appearance as that of fresh oil. Since the possible number of regeneration procedures is not regulated in any way, manufacturers of various regeneration units declare that the life of transformer oil can be extended indefinitely [4].

However, a study that compared the aging of fresh and regenerated oils under the influence of electrical discharges showed that the regenerated oil oxidizes much faster [5].

In this regard, it is necessary to pay attention to the degradation products formed in the oil during its aging in the power transformer. It is also necessary to consider in more detail the features of the procedure for cleaning oil from these compounds.

The hydrocarbon base of all transformer mineral oils is naphthenic and paraffinic compounds. The main reason for the degradation of the hydrocarbon base of oils is oxidation [2]. In this case, peroxides, alcohols, ketones, acids are formed. Also, in the process of thermal oxidative destruction in the oil, the content of polyaromatic and resinous-asphaltene compounds increases [6]. Based on the fact that not all molecules of the hydrocarbon base of the oil interact with oxygen, or turn into complex polyaromatic compounds, it is generally accepted that the removal of such compounds leads to the restoration of the hydrocarbon composition of the transformer oil [7].

The hydrocarbon molecules of all the considered degradation products of mineral oil have a polar character. To remove these aging products from oils, natural and artificial adsorbents are traditionally used. Those adsorbents have a developed surface and

* Corresponding author: g_marsels@mail.ru 
selectively absorb compounds of a polar nature [8, 9]. Natural sorbents are some varieties of clays, tripoli, opokas, diatomites, bauxites [10]. The most well-known examples are Zikeevsk Opoka and Fuller's Earth. Silica gel, alumina, aluminosilicate catalyst, etc. are traditionally used as synthetic adsorbents $[8,11]$.

The efficiency of the oil regeneration process is primarily associated with a decrease in the acid number. Therefore, it is the acid number that appears in most diagrams and tables illustrating the efficiency of regeneration by various sorbents. This approach is most likely due to the fact that the acid number in practice is considered one of the main markers of oil aging [8]. On the basis of acid number attempts are made to assess not only the condition, but also the resource of the oil [12]. In addition, the acid number is an obligatory standard indicator of the quality of commercial, fresh, operational, regenerated and stored oils [13].

However, the acid number, as well as the content of oxidation products in the oil, are not a complete characteristic of the degree of degradation of the hydrocarbon base of the oil. In addition to the aging products discussed above, unsaturated hydrocarbons are formed in the oil. Those unsaturated hydrocarbons are mainly with a $\mathrm{C}=\mathrm{C}$ double bond, as well as with a triple bond. Further, under unsaturated hydrocarbons in mineral transformer oils we mean compounds with a double $\mathrm{C}=\mathrm{C}$ bond that are alkenes and cycloalkenes.

Mineral oils are the products of refining crude oil. At the same time, in a number of oils from Eastern Siberia, Tatarstan and other regions of Russia, the content of alkenes can reach $15-20 \%$ of the oil mass [14]. Hydrocracking technology is used to remove unsaturated compounds from oil products at oil refineries. The main reason for the removal of unsaturated hydrocarbons is the increased reactivity of unsaturated bonds. The presence of a double bond in the carbon chain promotes its peroxidation [7]. In addition, unsaturated hydrocarbons are prone to polymerization, which contributes to increased sludge formation. Therefore, fresh transformer oils contain very little unsaturated hydrocarbons [7].

In view of the above, it seems relevant to study the processes of accumulation of unsaturated hydrocarbons in oils. The content of these compounds in transformer oil, unlike many other types of oil products, is currently not regulated. However, this parameter reflects the degree of degradation of the hydrocarbon base of mineral transformer oils.

The methods used in practice for the quantitative determination of the content of unsaturated hydrocarbons in liquid hydrocarbons are based on the reactions of addition of various substances to these hydrocarbons at the site of the double bond. The methods based on the reactions of iodine or bromine are the most widespread ones. The degree of unsaturation is estimated by the bromine or iodine number [15]. All these methods share all the disadvantages of traditional chemical analysis such as high labor intensity and duration of the analysis, the need to use chemical reagents, low accuracy and reproducibility of the analysis.
The alternative methods for determining the content of unsaturated hydrocarbons in oils are spectral methods, in particular, optical spectroscopy methods. This paper presents the results of determining the content of unsaturated compounds in mineral oils by IR absorption spectra.

\section{Experimental part}

Firstly, we studied the influence of the intensity of thermal oxidative destruction of mineral transformer oil on the change in the content of unsaturated compounds with a double bond $\mathrm{C}=\mathrm{C}$ in it. A decrease in the degree of thermal oxidation was modelled by reducing the initial concentration of an oxidation inhibitor (an additive ionol) in the oil. For the preparation of 4 samples, we used fresh base oil of the VG brand produced by Lukoil Oil Company (Russia), which does not contain ionol. The initial ionol concentration in the prepared oil samples was $0.2 \%, 0.1 \%, 0.05 \%$ and $0 \%$ respectively.

All samples were artificially aged in an oil bath in accordance with GOST 981-75 under the following conditions: temperature $-150^{\circ} \mathrm{C}$; oxidation time 14 hours; oxygen supply rate $-200 \mathrm{ml} / \mathrm{min}$; the catalyst is a copper plate.

As a result, samples of oxidized oils were obtained, designated as 1, 2, 3 and 4 in the Table 1.

Table 1. Description of oxidized oil samples.

\begin{tabular}{|c|c|c|}
\hline Sample no. & $\begin{array}{c}\text { Ionol } \\
\text { concentration } \\
\text { before } \\
\text { oxidation, \% }\end{array}$ & $\begin{array}{c}\text { Acid number, } \\
\text { mg KOH/g }\end{array}$ \\
\hline 1 & 0,20 & 0,02 \\
\hline 2 & 0,10 & 5,29 \\
\hline 3 & 0,05 & 19,59 \\
\hline 4 & 0 & 25,4 \\
\hline
\end{tabular}

In the second part of research, we determined the ability of artificial and natural adsorbents to remove unsaturated hydrocarbon molecules from mineral oils.

The following substances were used as artificial adsorbents:

a) silica gel KSKG (large-porous granular silica gel, produced by Salavat Catalyst Plant LLC, Sterlitamak, Russia), which is the main type of adsorbent used in practice in the regeneration of oils [16];

b) silica gel AS230SH (aluminosilicate adsorbent, produced by JSC "Chemical Plant named after L. Ya. Karpov", Mendeleevsk, Russia), recommended by leading Russian experts for the regeneration of transformer oils [17].

Three samples of oxidized transformer oils (samples I, II, and III) taken from power transformers were used 
for adsorption cleaning with the indicated silica gels. The procedure for adsorption cleaning of oil samples was carried out by settling the oils in a closed container filled with silica gel for 100 hours without stirring. The ratio of the mass of oil to the mass of silica gels was 2:1.

Oil samples purified with KSKG silica gel were designated as Ia, IIa, and IIIa. The oils purified with AS230SH silica gel were designated as Ib, IIb, and IIIb.

For all oil samples, the acid number was determined, the value of which is used to control the efficiency of the regeneration process [16]. Obtained results are presented in Table 2.

Fuller's Earth was used as a natural adsorbent. Adsorption cleaning was carried out by a regeneration unit manufactured by Globe Core [4] on the territory of an enterprise for the regeneration of used transformer oils (Yaroslavl region, Tutaev, Russia).

A sample of the used transformer oil (dark yellow, acid number - $0.02 \mathrm{mg} \mathrm{KOH} / \mathrm{g}$ ) supplied to the specified regeneration unit for treatment was designated as sample IV. The oil regenerated by Fuller's Earth was designated as sample IVc. As a result of regeneration, as in the case of silica gels, the acid number of the oil became less than $0.01 \mathrm{mg} \mathrm{KOH} / \mathrm{g}$.

The analysis of changes in the content of unsaturated compounds in all samples of oils was carried out according to the absorption intensity in the IR spectra of oils in the range of $4750 \div 4500 \mathrm{~cm}^{-1}$. As analytical bands we used absorption bands with peaks at about 4665 and $4600 \mathrm{~cm}^{-1}$, which belong to the vibrations of $=\mathrm{C}-\mathrm{H}$ and $\mathrm{C}=\mathrm{C}$ groups of unsaturated hydrocarbons [18]. IR spectra were obtained using a Tensor-27 (Bruker) IR-Fourier spectrometer with a spectral resolution of $1 \mathrm{~cm}^{-1}$. A $10-\mathrm{mm}$-thick cuvette was used, the window material was $\mathrm{CaF}_{2}$.

Fig. 1 shows the IR spectra of the optical density $D$ of oils samples number 1, 2, 3, and 4 from the Table 1 , as well as the spectrum of fresh VG oil, indicated in the figure as " 0 ".

Fig. 2 shows the IR spectra $D$ of old operating oils samples designated as I, II, III and IV, as well as the spectra of oils purified by various types of adsorbents.

\section{Results and discussion}

As can be seen from Table 1, the acid number of oils sequentially increases with decreasing initial ionol concentration. Thus, samples of oils number $1 \div 4$ represent a series with a consistently increasing degree of thermal destruction.

Analysis of the absorption spectra of these oils, presented in Fig. 1 shows that with an increase in the degree of thermal oxidation of oils, the intensity of absorption bands increases with peaks at 4665 and $4600 \mathrm{~cm}^{-1}$, which refer to unsaturated $\mathrm{C}=\mathrm{C}$ bonds. Thus, the hypothesis of an increase in amount of unsaturated hydrocarbons in mineral oil during its aging is confirmed.

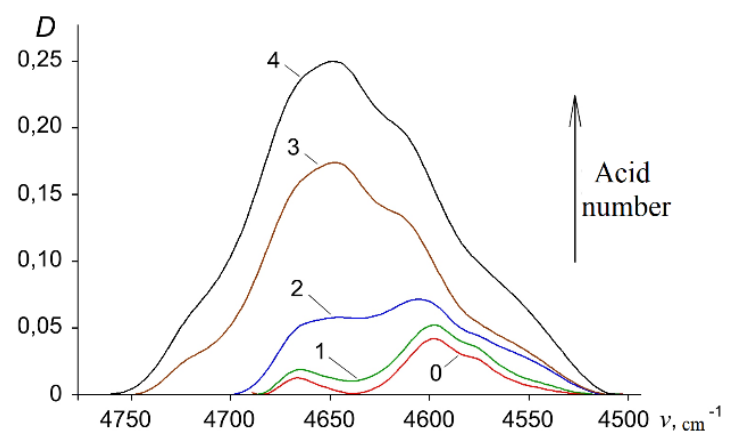

Fig. 1. Influence of the intensity of thermo-oxidative destruction on the change in absorption spectra of mineral oils.

Consequently, under real operating conditions, the formation of unsaturated bonds will continuously occur in the hydrocarbon molecules of the oil. And as the oils age, the content of such compounds will increase.

It should be noted that fresh oils also contain a certain amount of unsaturated hydrocarbons, as evidenced by the presence of these low intensity absorption bands in the sample " 0 " (fresh VG oil). We also note that the intensity of the absorption band with a peak at about $4665 \mathrm{~cm}^{-1}$ increases faster with an increase in thermal destruction than the intensity of the absorption band with a peak at $4600 \mathrm{~cm}^{-1}$.

Table 2. Characterization of the oxidation of oil samples.

\begin{tabular}{|c|c|c|c|c|c|}
\hline \multicolumn{2}{|c|}{ Base oils } & \multicolumn{2}{|c|}{ Purified with KSKG silica gel } & \multicolumn{2}{c|}{$\begin{array}{c}\text { Purified with AS230SH silica } \\
\text { gel }\end{array}$} \\
\hline Sample & $\begin{array}{c}\text { Acid number, } \\
\text { mg KOH/g }\end{array}$ & Sample & $\begin{array}{c}\text { Acid number, } \\
\text { mg KOH/g }\end{array}$ & Sample & $\begin{array}{c}\text { Acid number, } \\
\text { mg KOH/g }\end{array}$ \\
\hline I & 0,04 & Ia & $<0,01$ & Ib & $<0,01$ \\
\hline II & 0,08 & IIa & $<0,01$ & IIb & $<0,01$ \\
\hline III & 0,14 & IIIa & $<0,01$ & IIIb & $<0,01$ \\
\hline
\end{tabular}





Fig. 2. Absorption spectra of samples of mineral oils in operation and purified by various types of adsorbents: sample "0" - fresh VG oil. Types of adsorbents: a - KSKG; b - AS230SH; c - Fuller's Earth.

Such type of the $\mathrm{C}=\mathrm{C}$ absorption bands is the feature of unsaturated fats, the molecules of which contain carbonyl $\mathrm{C}=\mathrm{O}$ groups [19]. This feature in the spectra of strongly oxidized oils can be explained by the fact that, under the model conditions of accelerated thermal destruction of oils, two factors were combined: the bubbling of oils with oxygen and a low content of the oxidation inhibitor (ionol). In this case, most of the hydrocarbon molecules containing unsaturated bonds turned out to be oxidized. This is evidenced by the very high values of acid numbers of oil samples number 2,3 and 4 from Table 1 . Thus, the presence of $\mathrm{C}=\mathrm{O}$ groups in a significant part of unsaturated compounds led to a characteristic change in the ratio of the intensities of the absorption peaks.

Under real operating conditions, the degree of oxidation of the hydrocarbon base of oils is much lower $[7,20,21]$. Therefore, the peak at $4600 \mathrm{~cm}^{-1}$ is more intense. This can be seen from Fig. 2, which shows the spectra of oils I, II, III, and IV that have aged in power transformers for a long time.

As a result, after adsorption purification the acid number of all oils decreased to the limits of detection by the standard method (see Table 2). In addition, the color of the oils changed from dark brown to light yellow. This indicates the removal of most of the polyaromatic hydrocarbons (PAHs) from the oils. Thus, both silica gels and Fuller's Earth effectively remove polar compounds from the oil, which include organic acids and PAHs.

Comparison of the absorption spectra of oils purified with synthetic adsorbents with the spectra of crude oils shows that the intensity of absorption bands related to unsaturated hydrocarbons after purification decreases on average by less than $25 \%$. This allows us to state that after adsorption purification, the most part of the unsaturated compounds remains in the refined oils.

In Russian practice, most power transformers are equipped with thermosyphon or adsorption filters filled with KSKG adsorbents. Based on the results of the study, it can be argued that such devices are also not able to get rid of unsaturated hydrocarbons in oils.

In the process of the regeneration of oils by Fuller's Earth under factory conditions, the content of unsaturated compounds not only did not decrease, but also increased somewhat (see samples IV and IVc in Fig. 2). The obtained result can be explained by technological losses of oil in the regeneration unit, which are $10 \%$. Since unsaturated hydrocarbons in the bulk are non-polar compounds, their losses are insignificant.

Thus, during the operation of oil-filled transformers, the amount of unsaturated hydrocarbons in the oil is continuously increasing. This leads to a gradual decrease 
in the stability of the oil against oxidation, as well as an increase in the rate of sludge formation in the oil.

Based on the results obtained, it seems appropriate to monitor the content of unsaturated hydrocarbons in transformer oils. This parameter shows such a characteristic of the degree of degradation of the hydrocarbon base of oils, which cannot be restored by the methods of purification and regeneration used in practice. Obviously, when a certain amount of unsaturated hydrocarbons in the oil is reached, further operation of the oil becomes impractical and requires replacement with a new one.

For the development of appropriate criteria for the content of unsaturated compounds in transformer oils, it is most convenient to use the method of IR spectroscopy.

\section{Conclusion}

1. It has been proved for the first time that modern methods of purification and regeneration of transformer oils, based on the use of adsorption purification technology, are not able to completely remove unsaturated compounds from oils. This leads to a constant increase in amount of unsaturated compounds and deterioration of the operating properties of oils.

2. It is shown that the total content of unsaturated hydrocarbons is an indicator of the unrecoverable degree of degradation of the hydrocarbon base of mineral transformer oils. For the first time, a method for monitoring changes in the content of unsaturated hydrocarbons in mineral oils by their IR spectra in the range of $4750 \div 4500 \mathrm{~cm}^{-1}$ has been demonstrated.

\section{References}

1. J. Zou, W. Chen, F. Wan, Z. Fan, L Du, Energies, 9, 946 (2016).

2. P. Wiklund, B. Pahlavanpour, Journal of Iranian Association of Electrical and Electronics Engineers, 7, 65-73 (2010).

3. D.V. Shuvarin, Proceedings of scientific and practical conference 2015 Environmental safety of the energy sector: experience, problems, innovative solutions, 49-57 (2015).

4. CMM-R oil regeneration systems, (2019). https://globecore.com/products/oilregeneration/cmm-r-oil-regeneration-systems/ (Accessed 20 September 2020).

5. L. Safiddin, A. Boucherit, A.H.-Z. Zafour, I. Fofana, IET Generation Transmission \& Distribution, 12(21), 9 (2018).

6. O.A. Turanova, A.E. Vandjukov, V.K. Kozlov, A.N. Turanov, Optika i spektroskopija - Optics and spectroscopy, 114(4), 628-631.

7. R.A. Lipshtejn, M.I. Shahnovich, Transformer oil, 296 (1983).

8. I.V. Braj, Regeneration of transformer oils, 168 (1972).
9. V.M. Malyj, Proceedings of the $5^{\text {th }}$ International scientific and technical conference Electrical insulation, 192-199 (2010).

10. V.S. Ivanov, S.M. Fridman, Power engineering oils and lubricants, 280 (1972).

11. M.M. L'vova, V.B. Komarov, S.A. Kuljuhin, B.G. Ershov, Reliability and safety of power engineering, 2(29), 49-54 (2015).

12. S.P. Vysogorec, Reliability and safety of power engineering, 11(1), 41-47 (2018).

13. Rosseti, The scope and standards of testing electrical equipment, 262 (2017),

14. A.M. Syrkin, Je.M. Movsumzade, Fundamentals of Oil and Gas Chemistry, 109 (2002).

15. Z.A. Sablina, G.B. Shirokova, T.I. Ermakova, Laboratory methods for evaluating the properties of motor and jet fuels, 240 (1978).

16. STO 70238424.27.100.053-2013, Power oils and oil facilities of power plants and networks. Organization of operation and maintenance. Norms and requirements, 163 (2013).

17. I.R. Tatur, V.G. Spirkin, D.V. Shuvarin, A.V. Mel'nikov, D.V. Kurganov, Environmental protection in the oil and gas complex, 2, 17-21 (2017).

18. V.P. Krishhenko, Near infrared spectroscopy, 638 (1997).

19. M.Sh. Garifullin, V.K. Kozlov, Proceedings of the higher educational institutions. Energy sector problems, 9-10, 59-68 (2013).

20. V.A. Roginskij, Phenolic Antioxidants: Reactivity and Effectiveness, 247 (1988).

21. N.M. Jemmanujel', E.T. Denisov, Z.K. Majzus, Chain reactions of hydrocarbon oxidation in the liquid phase, 375 (1965). 